\title{
Resolution of statin-induced myalgia by correcting vitamin D deficiency: a case report
}

\begin{abstract}
The onset of myalgia is a well-known side effect of cholesterol-lowering statins. Recent studies advanced the hypothesis that reduced vitamin D levels may play a role in the onset of myalgia in statin users and potentially a vitamin D supplementation may be useful in these cases, as suggested by the present case report.

A 52-year-old man with a history of smoke and successfully controlled hypertension presented with chest pain and asthenia. He was diagnosed with stable angina pectoris and discharged on atorvastatin. Due to the onset of myalgia, the dosage of atorvastatin was reduced (from $40 \mathrm{mg}$ daily to $20 \mathrm{mg}$ daily) and then atorvastatin was switched to rosuvastatin without symptoms improvement. Switching from rosuvastatin to ezetimibe resulted in pain improvement, but also in plasma lipids increase beyond the normal range. Ezetimibe was switched to rosuvastatin + analgesic; in the meanwhile a high-performance liquid chromatography (HPLC) analysis showed low levels of 25-hydroxy-vitamin D and 1-25-dibydroxy-vitamin D. Therefore, vitamin $D_{3}$ was added to rosuvastatin, resulting in pain improvement, decrease of plasma lipids and progressive discontinuation of analgesics. During the follow-up, rosuvastatin was switched to atorvastatin + vitamin $D_{3}$, with a good control of plasma lipid levels and without the onset of myalgia.
\end{abstract}

Keywords: Myalgia; Statin; Vitamin D; Vitamin supplementation La mialgia durante il trattamento con statine può essere associata a bassi livelli plasmatici di vitamina $D$ e può migliorare dopo supplementazione vitaminica: un caso clinico CMI 2015; 9(1):21-26

bttp://dx.doi.org/10.7175/cmi.v9i1.978

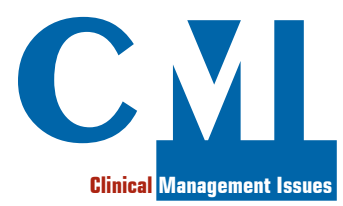

${ }^{1}$ Department of Health Science, University of Catanzaro and Operative Unit of Clinical Pharmacology and Pharmacovigilance, Azienda Ospedaliera MaterDomini, Catanzaro, Italy 2 Department of Neurology, "Annunziata" Hospital, Cosenza, Italy

\section{INTRODUCTION}

Treatment guidelines recommend LDL cholesterol lowering with statins as the primary means of cardiovascular risk reduction [1]. However, there is concern about the safety and tolerability of high-dose statin therapy. Indeed, adverse effects (AEs) on muscle (myopathy and, rarely, rhabdomyolysis) and liver (increased transaminases), while rare at standard doses, vary between statins and increase at higher doses [2].

We report the development of myalgia during statin treatment in a patient with low vitamin $\mathrm{D}$ levels, that improved after vitamin $\mathrm{D}$ supplementation.

\section{CASE REPORT}

In December 2012 a 52-year-old man presented with effort chest pain and as-

Why we describe this case

Myalgia is a well-known side effect of statins. Current guidelines suggest to evaluate creatinphosphokinase and transaminases before starting statin treatment, and this case report suggests to evaluate vitamin $D$ levels too. In statin users with low levels of vitamin $D, a$ supplementation may resolve muscle pain
Corresponding author Luca Gallelli Department of Health Science, University of Catanzaro, Viale Europa, 88100 Catanzaro

Operative Unit of Clinical Pharmacology and Pharmacovigilance,

Azienda Ospedaliera

MaterDomini,

Via Campanella 115,

Catanzaro, Italy.

Tel.: +390961712322

gallelli@unicz.it

Disclosure

The authors declare they have no competing financial interests concerning the topics of this article 
Table I. Laboratory findings

HVA = homovanillic acid; TSH $=$ thyroid-stimulating hormone; VAS = Visual Analogue Pain Score; $\mathrm{VMA}=$ vanilmandelic acid

\begin{tabular}{|c|c|c|c|c|c|c|}
\hline Parameter & $\begin{array}{l}\text { Normal } \\
\text { values }\end{array}$ & $\begin{array}{l}\text { Dec } \\
2012\end{array}$ & $\begin{array}{l}\text { Mar } \\
2013\end{array}$ & $\begin{array}{l}\text { Jun } \\
2013\end{array}$ & $\begin{array}{c}\text { Sep } \\
2013\end{array}$ & $\begin{array}{l}\text { Dec } \\
2014\end{array}$ \\
\hline VAS & & & 8 & 8 & 3 & 2 \\
\hline Glucose (mg/dl) & $70-100$ & 85 & 84 & & 90 & \\
\hline Creatinine clearance (ml/min) & $85-130$ & 92 & 94 & & 85 & \\
\hline Serum creatinine (mg/dl) & $0.7-1.2$ & 0.94 & 1 & & 1.1 & \\
\hline Potassium (mEq/l) & $3.6-5$ & 4 & 4.2 & 4.1 & 4 & \\
\hline Total cholesterol (mg/dl) & $<220$ & 452 & २२० & 210 & 280 & 210 \\
\hline LDL cholesterol (mg/dl) & $<130$ & 195 & 125 & 123 & 135 & 125 \\
\hline HDL cholesterol (mg/dl) & $35-39$ & 32 & 35 & 36 & 35 & 36 \\
\hline Triglyceride (mg/dl) & $50-150$ & 165 & 145 & & 145 & 145 \\
\hline 25-hydroxy-vitamin D (ng/ml) & 20-100 & & & & 12 & 35 \\
\hline 1-25-dihydroxy-vitamin D (ng/ml) & $25-66$ & & & & 19 & 42 \\
\hline Troponin I ( $\mu \mathrm{g} / \mathrm{l})$ & $<10$ & 0.25 & & & & \\
\hline Troponin T ( $\mu \mathrm{cg} / \mathrm{l})$ & $<0.1$ & 0.06 & & & & \\
\hline Creatinphosphokinase (IU/I) & $33-194$ & 95 & 350 & ४२० & 189 & 188 \\
\hline $\begin{array}{l}\text { Creatinphosphokinase myocardial } \\
\text { band (IU/I) }\end{array}$ & $0-25$ & 18 & 20 & 20 & 19 & 19 \\
\hline Aspartate aminotransferase (IU/I) & $8-48$ & 29 & 29 & 28 & 28 & 28 \\
\hline Alanine aminotransferase (IU/I) & $7-55$ & 28 & 28 & 29 & 28 & 28 \\
\hline Serum-free thyroxine (ng/dl) & $0.7-1.5$ & 0.9 & & & & \\
\hline TSH ( $\mu \mathrm{ll} / \mathrm{ml})$ & $0.1-4.5$ & 2.7 & & & & \\
\hline Urinary VMA (mg/day) & $1.8-6.7$ & 3.4 & & & & \\
\hline Urinary HVA (mg/day) & $0.5-6.2$ & 3.2 & & & & \\
\hline
\end{tabular}

thenia. Electrocardiography excluded the presence of pathological ST tract, and sublingual glyceryl trinitrate improved pain in about 4 minutes. Both coronary angiography and echocardiography were performed and did not shown any pathological heart disease.

The patient was a smoker (10 cigarettes/ day from the age of 22), with a 16-year history of hypertension successfully controlled $(135 / 85 \mathrm{mmHg})$ with valsartan plus hydrochlorothiazide ( $80 \mathrm{mg} / 12.5 \mathrm{mg} /$ day). Clinical examinations excluded the presence of a serious cardiac or respiratory disease (e.g. acute pericarditis, pneumonia, aortic dissection, acute coronary syndrome, Prinzmetal's angina) as well as gastroesophageal reflux. Blood chemical test performed at the admission and 6 hours later documented normal levels of cardiac troponin-I, troponin-T, creatinphosphokinase (CPK), CPK-myocardial band, thyroid-stimulating hormone and free thyroxine (Table I).

Routine blood assays documented high levels of cholesterol, LDL and triglycerides, whereas HDL cholesterol was low (Table I).
The evaluation of urinary catecholamines revealed normal values (Table I).

A diagnosis of stable angina pectoris was postulated and the patient was discharged on atorvastatin $40 \mathrm{mg} /$ day.

About 3 months later, during the followup, blood chemical tests revealed a decrease in plasma lipids, but the patient referred the onset of myalgia (visual analogue pain score, VAS = 8).

The clinical history revealed that no other drugs or other substances were taken during this period; moreover, the patient didn't perform job or physical activity that could be responsible for the muscular pain.

Physical examination documented a diffuse, reproducible pain with palpation or manipulation of upper and lower extremities without evidence of joint effusion or redness. Doppler ultrasound excluded the presence of peripheral vascular diseases, while laboratory findings excluded the presence of hepatic, renal or gastrointestinal diseases.

Naranjo probability scale [3] documented a possible association between statin and muscular pain, therefore the dosage of ator- 


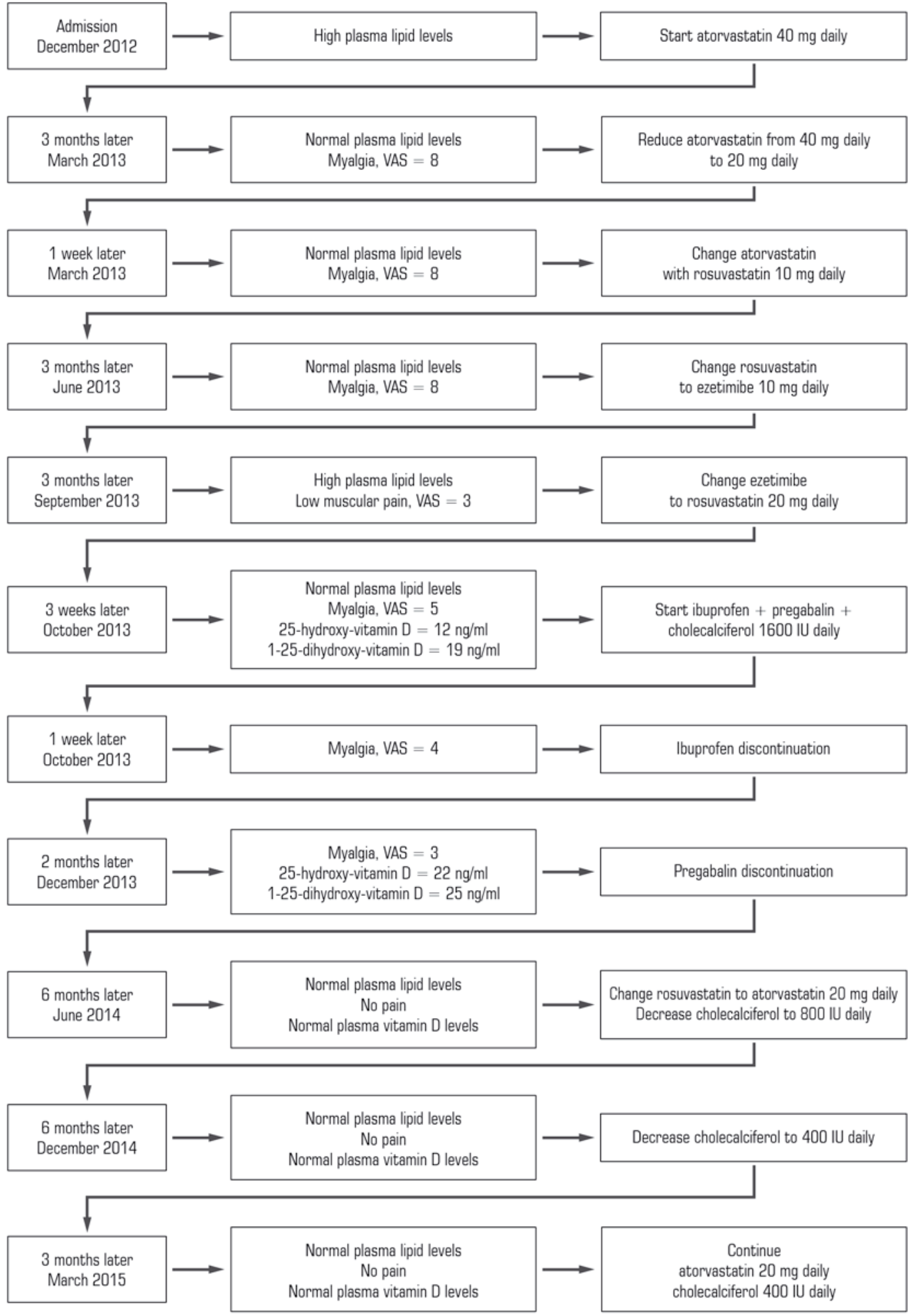

vastatin was reduced to $20 \mathrm{mg} /$ day. About one week later the patient came back for the persistence of muscular pain $(\mathrm{VAS}=8)$, self treated with non-steroidal antinflammatory drugs, and atorvastatin $(20 \mathrm{mg} /$ day $)$ was switched to rosuvastatin $(10 \mathrm{mg} /$ day $)$. Three months later, at the follow-up, biochemical tests revealed high levels of CPK and the patient complained about an intense pain $(\mathrm{VAS}=8$; Table I), therefore rosuvastatin was stopped and ezetimibe $(10 \mathrm{mg} /$ day $)$ was started. After three additional months,
Figure 1. Time course of drug treatment during the study 
at the follow-up, the patient showed an improvement in muscular pain (VAS $=3$ ), but laboratory analysis revealed an increase in plasma lipids (Table I).

Therefore, ezetimibe was stopped and rosuvastatin $(20 \mathrm{mg} /$ day $)$ was started; a normalization of biochemical values occurred in three weeks, but with the onset of muscular pain $(V A S=5)$. In order to reduce the pain, a treatment with ibuprofen (600 $\mathrm{mg}$ daily) + pregabalin (75 mg daily) was prescribed and plasma vitamin D levels were evaluated.

Using a reversed phase (C18) High-Performance Liquid Chromatography system (HPLC; UV detector $265 \mathrm{~nm}$, limit of detection $1.5 \mathrm{ng} / \mathrm{ml}$; intra-assay and inter-assay coefficients of variation for control: $8 \%$ ), we documented low levels of 25-hydroxyvitamin $\mathrm{D}$ and of 1-25-dihydroxy-vitamin D (Table I). Clinical evaluation revealed that low plasma vitamin $\mathrm{D}$ levels were related to inadequate vitamin $\mathrm{D}$ intake, and a daily treatment with cholecalciferol (Vita$\min \mathrm{D}_{3}, 1600 \mathrm{IU}$ daily) taken during a fatcontaining meal was prescribed and after 1 week the patient referred an improvement of muscular pain (VAS $=4$ ), ibuprofen was dismissed and the patient was maintained on treatment with vitamin $\mathrm{D}_{3}+$ rosuvastatin + pregabalin.

Two months later, the patient referred an improvement of pain (VAS = 3) and laboratory findings revealed a decrease of plasma lipid levels and an increase in both 25-hydroxy-vitamin D (22 ng/ml) and 1-25-dihydroxy-vitamin D $(25 \mathrm{ng} / \mathrm{ml})$. Pregabalin was dismissed and 6 months later the patient referred the absence of muscular pain, while laboratory tests revealed a normalization of plasma lipids. Rosuvastatin was changed to atorvastatin $(20 \mathrm{mg} /$ day $)$ and cholecalciferol dosage was decreased (800 mg daily).

Six months later (December 2014), during the follow-up, laboratory findings revealed normal levels of both lipids and vitamins D, therefore cholecalciferol dosage was reduced to 400 IU daily.

Main questions a doctor should ask him/herself in this situation

- What is the patient's job?

- How is the patient's diet?

- Are there other drugs that could induce myalgia (e.g. corticosteroids) or statin toxicity (e.g. CYP3A4 or CYP2C9 inbibitors)?

- Have I evaluated the development of myalgia?

- Have I evaluated the values of vitamins D before administering statins?
To date, on March 2015, the patient is on atorvastatin therapy $(20 \mathrm{mg} /$ day $)$ and on vitamin D supplementation (cholecalciferol 400 IU daily), laboratory findings are in normal range and no side effects are recorded (Figure 1).

\section{DISCUSSION}

We report the improvement of statin-induced myalgia through vitamin $\mathrm{D}$ supplementation.

Previously, we documented that drugdrug interaction is common during drug treatment [4,5] and Settergren et al. [6] during a cross-sectional analysis of nationwide dispensing data documented that calcium blockers and gemfibrozil can induce a drug-drug interaction with statins, with the development of myalgia.

In the present case, myalgia appeared during the treatment with either atorvastatin or rosuvastatin, both metabolized by cytochrome P450 (CYP), CYP3A4 and CYP2C9, respectively. Our patient did not take any drug able to cause a CYP inhibition, therefore the drug-drug interaction was ruled out. Moreover, musculoskeletal pain occurred after the beginning of atorvastatin therapy and increased in a timedependent manner. The decrease of atorvastatin dosage (from 40 to $20 \mathrm{mg}$ ) and then the switch to rosuvastatin $(10 \mathrm{mg})$ did not improve muscular pain. Clinical and laboratory assays confirmed the myalgia and excluded the presence of systemic diseases able to induce the development of muscular disease. In agreement with our previous papers $[7,8]$, using the Naranjo score, we documented a probable association between statins and clinical symptoms. Therefore, following guidelines recommendations [1], rosuvastatin was changed to ezetimibe with a decrease of muscular pain but with an increase in plasma lipids. The re-challenge of rosuvastatin, after the discontinuation of ezetimibe, induced the normalization of lipid levels but the development of myalgia, that was treated with pregabalin and with ibuprofen as needed.

Recently, an association between myalgia in statin treated patients and low vitamin $\mathrm{D}$ plasma levels has been reported [9-12].

In particular, Morioka and coworkers [9], using the National Health and Nutrition Examination Survey 2001-2004, performed a cross-sectional study on statin users and 
evidenced that vitamin D plasma concentrations were significantly lower in patients with statin-associated myalgia compared with patients without muscle pain.

In agreement, Michalska-Kasiczak and coworkers [10] evaluating 437 articles from 1987 to 2014 documented an association between low vitamin D levels (mean $28.4 \pm 13.80 \mathrm{ng} / \mathrm{ml}$, normal range $34.86 \pm 11.63 \mathrm{ng} / \mathrm{ml}$ ) and myalgia in patients on statin therapy.

In contrast, evaluating 10,001 patients with stable coronary heart disease randomized to atorvastatin $80 \mathrm{mg}$ vs $10 \mathrm{mg}$ daily, Bittner and coworkers in a poster presentation did not shown any relationship between vitamin $\mathrm{D}$ deficiency and myalgia incidence [13].

A recent retrospective study postulated that the correction of 25-hydroxy-vitamin D levels before statin therapy initiation may mitigate the development of statin-related myalgia [14].

Accordingly, in the present case we recorded low plasma vitamin $\mathrm{D}$ levels, that have probably increased the statin-muscle toxicity.

Previously, Draeger and coworkers [15], using an electron microscope, documented, in skeletal muscle biopsies from statin-treated patients, the presence of a breakdown of the T-tubular system and subsarcolemmal rupture that were not present in muscle biopsies of non-treated-patients.

In addition, in an experimental study performed in muscles of vitamin $\mathrm{D}$ depleted rabbits, Pfeifer and coworkers [16] documented a decrease of ATP-dependent calcium uptake of isolated vesicles in the sarcoplasmic reticulum. Moreover, they documented that $1,25(\mathrm{OH})_{2} \mathrm{D}$ plays a role in the active transportation of calcium into sarcoplasmic reticulum and increases the intracellular levels of ATP and phosphate increasing protein synthesis.

Taken together, these data suggest that vitamin $\mathrm{D}$ is involved in the maintenance of normal muscle physiology and low levels of vitamin $\mathrm{D}$ can induce a muscle damage impairing physical symptoms during the treatment with statins.

Al-Said et al. [17] demonstrated reversible electromyographic changes, consistent with a myopathy, that subsequently resolved once the vitamin $\mathrm{D}$ deficits $(<20 \mathrm{ng} / \mathrm{ml})$ were resolved.

Binkley et al. [18], evaluating the effects of daily and once monthly dosing of ergocalciferol or cholecalciferol on circulating 25-hydroxy-vitamin D, documented that the administration of cholecalciferol is able to induce a normalization of plasma vitamin $\mathrm{D}$ levels at both daily and one monthly dosage.

Recently, it has been reported that the usual supplement dose for vitamin D deficiency is 1000 international units (vitamin $\mathrm{D}_{3}$ ) daily, vitamin $\mathrm{D}_{2}$ supplementation at doses of 50,000 international units can be given for correction [19]. However, in particular for vitamin $\mathrm{D}_{2}$ supplementation, an increased risk of mortality for cardiovascular disease was observed [20].

In our case low levels of vitamin $\mathrm{D}$ were related to inadequate intake, therefore vitamin $\mathrm{D}_{3}$ supplementation during a fat meal and the treatment with statin induced the normalization of plasma vitamin D levels and lipid levels without the development of statin-related muscular side effects.

In agreement with the indication of Italian Agency for Drug (Agenzia Italiana del Farmaco-AIFA), in Italy rosuvastatin may be prescribed only when other statins cannot be used (second choice), therefore the absence of pain induced the change of rosuvastatin to atorvastatin $20 \mathrm{mg}$ daily with a good control of blood lipids.

In conclusion, in this report we documented the improvement of muscular pain through a supplementation of vitamin D in a patient treated with statins. However, this observation should be corroborated by further studies involving large groups of patients.

Keypoints

- The onset of myalgia is a well-known side effect of statins

- However a thorough examination should be performed to rule out other causes of myalgia in statin users

- In the presence of muscle pain, we suggest to check also vitamin $D$ levels

- In this patient vitamin D supplementation was effective in muscular pain reduction during statin treatment 


\section{REFERENCES}

1. Stone NJ, Robinson J, Lichtenstein AH, et al. 2013 ACC/AHA guideline on the treatment of blood cholesterol to reduce atherosclerotic cardiovascular risk in adults: a report of the American College of Cardiology/American Heart Association Task Force on Practice Guidelines. J Am Coll Cardiol 2014; 63: 2889-934; http://dx.doi.org/10.1016/j.jacc.2013.11.002

2. Armitage J. The safety of statins in clinical practice. Lancet 2007; 370: 1781-90; http://dx.doi. org/10.1016/S0140-6736(07)60716-8

3. Naranjo CA, Busto U, Sellers EM, et al. A method for estimating the probability of adverse drug reactions. Clin Pharmacol Ther 1981; 30: 239-45; http://dx.doi.org/10.1038/clpt.1981.154

4. Palleria $\mathrm{C}, \mathrm{Di}$ Paolo $\mathrm{A}$, Giofrè $\mathrm{C}$, et al. Pharmacokinetic drug-drug interaction and their implication in clinical management. J Res Med Sci 2013; 18: 601-10

5. Gallelli L, Ferraro M, Spagnuolo V, et al. Rosuvastatin-induced rhabdomyolysis probably via CYP2C9 saturation. Drug Metabol Drug Interact 2009; 24: 83-7; http://dx.doi.org/10.1515/ DMDI.2009.24.1.83

6. Settergren J, Eiermann B, Mannheimer B. Adherence to drug label recommendations for avoiding drug interactions causing statin-induced myopathy-A Nationwide Register Study. PLoS ONE 2013; 8: e69545; http://dx.doi.org/10.1371/journal.pone.0069545

7. De Vuono A, Palleria C, Scicchitano F, et al. Skin rash during treatment with generic itraconazole. J Pharmacol Pharmacother 2014; 5: 158-60; http://dx.doi.org/10.4103/0976-500X.130086

8. Mumoli L, Gambardella A, Labate A, et al. Rosacea-like facial rash related to metformin administration in a young woman. BMC Pharmacol Toxicol 2014; 15: 3; http://dx.doi. org/10.1186/2050-6511-15-3

9. Morioka TY, Lee AJ, Bertisch S, et al. Vitamin D status modifies the association between statin use and musculoskeletal pain: A population based study. Atherosclerosis 2014; 238: 77-82; http:// dx.doi.org/10.1016/j.atherosclerosis.2014.11.012

10. Michalska-Kasiczak M, Sahebkar A, Mikhailidis DP, et al. Analysis of vitamin D levels in patients with and without statin-associated myalgia - A systematic review and meta-analysis of 7 studies with 2420 patients. Int J Cardiol 2014; 178C: 111-6; http://dx.doi.org/10.1016/j. ijcard.2014.10.118

11. Duell PB, Connor WE. Vitamin D deficiency is associatedwith myalgias in hyperlipidemic subjects taking statins. Circulation 2008; 118: 470

12. Ahmed W, Khan N, Glueck CJ, et al. Low serum $25(\mathrm{OH})$ vitamin D levels $(<32 \mathrm{ng} / \mathrm{mL})$ are associated with reversible myositis-myalgia in statin-treated patients. Transl Res 2009; 153: 11 6; http://dx.doi.org/10.1016/j.trsl.2008.11.002

13. Bittner V,Wenger NK, Waters DD, et al. Vitamin D levels are not related to myalgias in statintreated patients with stable coronary disease. J Am Coll Cardiol 2010; 55: 177-1659 (poster contribution); http://dx.doi.org/10.1016/S0735-1097(10)61571-9; http://dx.doi.org/10.1016/ S0735-1097(10)61660-9

14. Mergenhagen K, Ott M, Heckman K, et al. Low vitamin D as a risk factor for the development of myalgia in patients taking high-dose simvastatin: a retrospective review. Clin Ther 2014; 36: 770-7; http://dx.doi.org/10.1016/j.clinthera.2014.02.023

15. Draeger A, Monastyrskaya K, Mohaupt M, et al. Statin therapy induces ultrastructural damage in skeletal muscle in patients without myalgia. J Pathol 2006; 210: 94-102; http://dx.doi. org/10.1002/path.2018

16. Pfeifer M, Begerow B, Minne HW. Vitamin D and muscle function. Osteoporos Int 2002; 13: 187-94; http://dx.doi.org/10.1007/s001980200012

17. Al-Said YA, Al-Rached HS, Al-Qahtani HA, et al. Severe proximal myopathy with remarkable recovery after vitamin D treatment. Can JNeurol Sci 2009; 36: 336-9; http://dx.doi.org/10.1017/ S0317167100007083

18. Binkley N, Gemar D, Engelke J, et al. Evaluation of ergocalciferol or cholecalciferol dosing, 1,600 IU daily or 50,000 IU monthly in older adults.J Clin Endocrinol Metab 2011; 96: 981-8; http://dx.doi.org/10.1210/jc.2010-0015

19. Chaney A. Primary care management of vitamin D deficiency and liver disease. J Am Assoc Nurse Pract 2015 Feb 26 [Epub ahead of print]; http://dx.doi.org/10.1002/2327-6924.12217

20. Chowdhury R, Kunutsor S, Vitezova A, et al. Vitamin D and risk of cause specific death: systematic review and meta-analysis of observational cohort and randomised intervention studies. BMJ 2014; 348: g1903; http://dx.doi.org/10.1136/bmj.g1903 